\title{
Prevalence of Risk Factors for Adverse Pregnancy Outcomes During Pregnancy and the Preconception Period-United States, 2002-2004
}

\author{
John E. Anderson · Shahul Ebrahim • Louise Floyd • \\ Hani Atrash
}

Published online: 19 May 2006

(C) Springer Science+Business Media, Inc. 2006

\begin{abstract}
Objectives: To assess the prevalence of risk factors for adverse pregnancy outcome during the preconception stage and during pregnancy, and to assess differences between women in preconception and pregnancy. Methods: Data from the 2002 and 2004 Behavioral Risk Factor Surveillance System, United States, were used to estimate the prevalence of selected risk factors among women 18-44 in the preconception period (women who wanted a baby in the next 12 months, and were not using contraception, not sterile and not already pregnant) with women who reported that they were pregnant at the time of interview. Results: Major health risks were reported by substantial proportions of women in the preconceptional period and were also reported by many pregnant women, although pregnant women tended to report lower levels of risk than preconception women. For example, $54.5 \%$ of preconception women reported one or more of 3 risk factors (frequent drinking, current smoking, and absence of an HIV test), compared with $32.0 \%$ of pregnant women $(p<.05)$. The difference in the prevalence of these three risk factors between preconception and pregnancy was significant for women with health insurance $(52.5 \%$ in preconception vs. $29.4 \%$ in pregnancy, $p<.05)$, but not for women without insurance (63.4\% vs. $52.7 \%, p>.05)$. Conclusions: Women appear to be responding to messages regarding behaviors that directly affect pregnancy such as smoking, alcohol con-
\end{abstract}

J. E. Anderson $(\square)$

Division of Reproductive Health/K-22, Centers for Disease

Control and Prevention,

Atlanta, GA 30333

e-mail: jea1@cdc.gov

S. Ebrahim · L. Floyd $\cdot$ H. Atrash

Division of Birth Defects and Developmental Disabilities, Centers for Disease Control and Prevention,

Atlanta, GA 30333 sumption and taking folic acid, but many remain unaware of the benefits of available interventions to prevent HIV transmission and birth defects. Although it appears that some women reduce their risk for adverse pregnancy outcomes after learning of their pregnancy, the data suggest that a substantial proportion of women do not. Furthermore, if such change occurs it is often too late to affect outcomes, such as birth defects resulting from alcohol consumption during the periconception period. Preconception interventions are recommended to achieve a more significant reduction in risk and further improvement in perinatal outcomes.

Keywords Pregnancy $\cdot$ Riskfactors · Preconception

\section{Introduction}

Although the US has achieved substantial gains in improving the health of women and children, and infant mortality has reached record low levels, the US presently ranks 27 th among established market economies in infant mortality [1]. Four recurring causes account for more than half of all infant deaths: birth defects, disorders related to short gestation and preterm birth, maternal complications of pregnancy (including complications of the placenta, cord and membranes), and sudden infant death syndrome [2]. In recent years some causes of infant mortality have increased, particularly in the percentage of births that were preterm and of low birth weight [3]. In 2002, congenital anomalies, low birth weight, preterm delivery, and maternal complications of pregnancy accounted for 14,263 (50.9\%) of the 28,034 infant deaths [2].

Relatively little is known about the risk factors underlying the continued increase in these adverse outcomes. Adequate prenatal care has long been considered as an opportunity to 
reduce such risks. However, despite increases in access to and utilization of early prenatal care, interventions and efforts directed at addressing such risk factors fall short of their goal. Indeed, the effects of such efforts may have reached their peak, and new approaches may be necessary. Reviews on selected risk factors indicate that a large proportion of women enter pregnancy with pre-existing risks for adverse pregnancy outcomes. Although some women tend to take action to reduce their risk as soon as they learn that they are pregnant, the extent of pregnancy related change in risk factors varies considerably and often does not occur early in pregnancy when teratogenic effects are more pronounced. Moreover, post-pregnancy relapse is high [4]. For example in the period 1996-1998 the reported reduction in the use of alcohol, tobacco and illicit drugs in the first trimester was 46,28 , and $28 \%$ respectively [4]. Such information is important to the emerging emphasis on preconception care as a complementary approach to reduce risks to pregnancy. This paper provides nationally-representative estimates on risks during the preconception period and describes the apparent reductions in risk achieved during pregnancy for all known risk factors for which data are available.

\section{Data}

The estimates presented herein are based on data from the 2004 Behavioral Risk Factor Surveillance System (BRFSS) [5]. However, because not all risk indicators that were considered were included in the 2004 BRFSS, 2002 data were used for a small number of risk indicators for which the 2002 BRFSS provided the most recently available data. The BRFSS is an ongoing annual telephone survey of the non-institutionalized adult civilian population aged 18 years and older conducted in each state. The survey obtains information on a wide range of modifiable risk behaviors. In 2004 the median response rate for the BRFSS state surveys was 52.7 percent. This rate represents an estimate of the percentage of eligible respondents that completed telephone interviews, and is computed based on procedures recommended by the Council of American Survey Research Organizations (http://www.casro.org/resprates.cfm.) In comparison with other national surveys, BRFSS data appear to be of good quality [5]. Additional technical information is available online at http://www.cdc.gov/brfss/.

The survey includes core modules asked in all states, rotating modules asked every second year in all states, and optional modules that are included only in some states. Being a general survey, the BRFSS lacks information on some factors of interest to the present topic, for example month of pregnancy for pregnant women, or information on prenatal care. Further, the BRFSS is not designed specifically to study all known risk behaviors at preconception or pregnancy. In addition, as an interview survey, the survey relies on selfreported data which contain an unknown level of reporting error.

Identification of women in the preconception group in the BRFSS is made possible by questions introduced in the "family planning section" of the questionnaire. The availability of these questions makes it possible to compare risk behaviors among women about to become pregnant with those who are already pregnant on a large and nationally-representative sample of women. Women in the preconception period were identified as those who reported that they wanted a baby in the next 12 months, were not using contraception, were not sterile and were not already pregnant. Women who were pregnant were identified based on the response to the question "To your knowledge are you now pregnant?" Age of gestation was not assessed in this survey. Data were aggregated across states to make national estimates. For 2004 this resulted in a total national U.S. sample of 70,917 women aged 18 to 44 years, of whom 2308 (3.4\%) were classified into the preconception period, and 2998 (4.7\%) reported that they were pregnant at the time of their interview. The corresponding number of women in the 2002 are 61,284 women $18-44$, with $2204(3.7 \%)$ in the preconception period, and $2556(4.6 \%)$ pregnant women. The analysis on folic acid and vitamins are based on surveys in 12 states in 2004. The results for these items are generalizable to the populations of these 12 states (shown in Table 2) but not to the entire United States population.

\section{Statistical methods}

The percentage of women exposed to 21 risk indicators was identified for women in 2 groups: women in the preconception period, and pregnant women (Table 1). The categories of risk indicators for which data are available include perceived general and mental health, lack of medical insurance, knowledge of HIV prevention and practices, alcohol and tobacco use, obesity, nutrition including folic acid uptake for the prevention of birth defects, and presence of chronic medical conditions.

Because these are not longitudinal data it was not possible to observe behavior change in individual women. Rather the prevalence of risk factors was compared between preconception and pregnant women as a proxy measure of pregnancyrelated risk reduction [6], although is is possible that other factors could influence the differences between preconception and pregnant women. The statistical significance between preconception and pregnant women of the percentage reporting each risk factor, was assessed using a $t$-test. All statistical analysis was based on weighting factors designed 
Table 1 Comparison of age and race/ethnicity, 2004 Women in preconception and pregnant (BRFSS) and Births (Vital Statistics data)

\begin{tabular}{|c|c|c|c|c|c|}
\hline & \multicolumn{5}{|l|}{ BRFSS } \\
\hline & \multicolumn{2}{|c|}{ Preconception women } & \multicolumn{2}{|c|}{ Pregnant women } & \multirow{2}{*}{$\begin{array}{l}\text { Vital statistics } \\
\text { births Percent }\end{array}$} \\
\hline & Percent & $+/-\mathrm{CI}$ & Percent & $+/-\mathrm{CI}$ & \\
\hline \multicolumn{6}{|l|}{ Age group } \\
\hline $18-19$ & 2.0 & 0.9 & 7.5 & 2.2 & 7.1 \\
\hline $20-24$ & 16.3 & 3.3 & 26.5 & 3.0 & 26.1 \\
\hline $25-29$ & 23.4 & 3.1 & 26.5 & 2.7 & 27.9 \\
\hline $30-34$ & 28.4 & 3.1 & 26.2 & 2.8 & 24.4 \\
\hline $35-39$ & 18.5 & 2.5 & 10.2 & 1.5 & 12.0 \\
\hline \multirow[t]{2}{*}{$40-44$} & 11.4 & 2.2 & 3.1 & 0.9 & 2.6 \\
\hline & 100.0 & & 100.0 & & \\
\hline \multicolumn{6}{|l|}{ Race/ethnicity } \\
\hline NH White & 64.9 & 3.9 & 60.1 & 3.4 & 56.8 \\
\hline NH Black & 11.9 & 2.5 & 11.5 & 1.9 & 13.6 \\
\hline Hispanic & 16.0 & 3.5 & 23.0 & 3.4 & 22.5 \\
\hline \multirow[t]{2}{*}{ Others } & 7.2 & 2.1 & 5.5 & 1.6 & 7.2 \\
\hline & 100.0 & & 100.0 & & 100.0 \\
\hline
\end{tabular}

Note. For BRFSS estimates, 95\% confidence interval = Percent $+/-$ CI. Vital statistics data: Hamilton BE, Martin JA, Ventura SJ, Sutton PD, Menacker F. Births: Preliminary data for 2004. National vital statistics reports; vol 54 no 8. Hyattsville, Maryland: National Center for Health Statistics. 2005.

to produce unbiased estimates, and statistical tests were adjusted for complex sample design using the software for survey data analysis (SUDAAN, Research Triangle Institute, Research Triangle Park, NC).

\section{Results}

Comparisons with vital statistics data on births in 2004 indicate that the 2004 BRFSS respondents in the preconception stage and pregnant when interviewed were distributed very similarly by age and race-ethnicity to women giving birth in 2004 (Table 1). Overall, for many risk factors, pregnant women reported lower prevalence of risk than preconception women (Table 2). Percentages reporting alcohol consumption and smoking were much lower for pregnant women than preconception women. For example, $53.9 \%$ of preconception women reported any use of alcohol in the past month, compared with $10.7 \%$ of pregnant women, $19.4 \%$ of preconception women reported current smoking in contrast to $8.4 \%$ of pregnant women, and, in the 12 states that included the question, $44.8 \%$ of preconception women reported not taking vitamins with folic acid versus $19.9 \%$ of pregnant women.

For some risk indicators, no statistically significant difference was observed between women in the preconception period and pregnant women. The lack of awareness about methods to prevent mother-to-child HIV transmission (PMTCT) was not significantly different between preconception women and pregnant women $(38.2 \%$ vs. $34.1 \%)$. However, a small but significantly higher percentage of pregnant women reported HIV risk (6.9\%) compared to preconception women $(4.3 \%)$, which is perhaps related to sexual risk behaviors being causally linked to both HIV and to unplanned pregnancies.

Of concern, is that although a lower percentage of pregnant women compared with preconception women had never been tested for HIV, about one-fourth of pregnant women remained untested, despite national recommendations that call for all pregnant women to be tested for HIV [7].

In the case of chronic diseases such as diabetes, the lower level of disease among pregnant women may indicate selfselection of diabetic women to avoid pregnancy. Of note, nearly one fifth of the women in preconception period were obese (defined as having a body mass index or BMI of greater than 30) and twice that number were overweight (BMI of greater than 25) (Table 2). Most preconception and pregnant women did not report consuming the recommended 5 servings of fruits and vegetables per day [8], although the percentage was lower for pregnant women $(64.7 \%)$ compared with women in the preconception period $(74.9 \%)$. Indicators of general well being and recent dental care were not different between women in preconception period and pregnancy.

A composite measure of 3 basic pregnancy risks was formed, frequent alcohol consumption, current smoking and lack of an HIV test, in order to use the BRFSS data to illustrate the size of the target populations for preconception care. These are risk factors for which we have data that are generalizable to the US population, and for which interventions or information are widely available or promoted. The results indicate that $54.5 \%$ of preconception women reported one or 
Table 2 Health risk indicators by pregnancy status Women 18-44, 2004 and 2002 BRFSS

\begin{tabular}{|c|c|c|c|c|}
\hline & \multicolumn{2}{|c|}{ Preconception women } & \multicolumn{2}{|c|}{ Pregnant women } \\
\hline & Percent & $+/-\mathrm{CI}$ & Percent & $+/-\mathrm{CI}$ \\
\hline \multicolumn{5}{|l|}{2004 data-nationwide ${ }^{a}$} \\
\hline \multicolumn{5}{|l|}{ General Health } \\
\hline Poor/fair general health status & 8.3 & 2.1 & 6.4 & 1.7 \\
\hline $14+$ days in past month mental health not good* & 12.8 & 2.4 & 9.6 & 1.9 \\
\hline No health plan* & 18.8 & 3.5 & 11.9 & 2.3 \\
\hline No dental visit past year & 28.3 & 3.2 & 30.2 & 3.1 \\
\hline Told had diabetes* & 2.0 & 0.9 & 0.7 & 0.4 \\
\hline \multicolumn{5}{|l|}{ HIV } \\
\hline Don't know about prevention of MTC HIV transmission & 38.2 & 3.5 & 34.1 & 3.0 \\
\hline Never tested for HIV* & 34.9 & 3.4 & 24.4 & 3.0 \\
\hline 1 or more HIV risk category* & 4.3 & 1.4 & 6.9 & 1.8 \\
\hline \multicolumn{5}{|l|}{ Alcohol/smoking } \\
\hline Any alcohol in past month* & 53.9 & 3.7 & 10.7 & 1.8 \\
\hline Average 1 or more drink per day, past month* & 6.0 & 2.0 & 0.8 & 0.5 \\
\hline Binging: Any occasions of $5+$ drinks in past month* & 10.7 & 2.1 & 1.9 & 0.9 \\
\hline Frequent drinking: binging or 1 or more drinks per day* & 12.9 & 2.5 & 2.2 & 0.9 \\
\hline Current smoker* & 19.4 & 2.7 & 8.4 & 1.5 \\
\hline \multicolumn{5}{|l|}{ Obesity } \\
\hline Overweight, body mass index $(\mathrm{BMI})>25$ & 46.0 & 3.6 & NA & \\
\hline Obese, $\mathrm{BMI}>30$ & 22.4 & 3.1 & NA & \\
\hline \multicolumn{5}{|l|}{2004 data, 12 states only ${ }^{b, c}$} \\
\hline \multicolumn{5}{|l|}{ Folic acid } \\
\hline Don’t know about folic acid for birth defects prevention & 46.1 & 6.9 & 38.4 & 5.6 \\
\hline Don't take vitamins of any kind* & 36.9 & 7.1 & 10.2 & 3.6 \\
\hline Don't take folic acid or multivitamin* & 38.4 & 7.0 & 17.5 & 4.5 \\
\hline Don't take folic acid or multivitamin daily* & 44.8 & 6.9 & 19.9 & 4.7 \\
\hline \multicolumn{5}{|l|}{ Nutrition $\left(2002\right.$ BRFSS) ${ }^{\mathrm{d}}$} \\
\hline Fewer than 5 servings/fruit and vegetables* & 74.9 & 3.1 & 64.7 & 3.5 \\
\hline Fewer than 1 servings/fruit and vegetables & 3.3 & 1.1 & 2.9 & 1.3 \\
\hline
\end{tabular}

Note. $95 \%$ confidence interval $=$ Percent $+/-\mathrm{CI}$.

${ }^{a}$ Unweighted number of observations: Preconception women (2308), Pregnant Women (2998).

${ }^{b}$ Unweighted number of observations: Preconception women (607), Pregnant Women (756).

${ }^{c}$ States: AZ, CO, FL, KY, MN, MT, NC, ND, NE, TX, VA, WI.

${ }^{d}$ Unweighted number of observations: Preconception women (2204), Pregnant Women (2556).

${ }^{*} p<.05$, preconception vs. pregnant women.

**HIV risk: Any statement is true: in past year illegal drug injection, treated for STD, exchange for money/drugs, uprotected anal intercourse.

Table 3 Percentage and estimated number reporting any of 3 risks for pregnancy outcome ${ }^{a}$ for preconception and pregnant women aged 18-44 Women 18-44, 2004 BRFSS

\begin{tabular}{|c|c|c|c|c|c|c|c|c|c|c|}
\hline & \multicolumn{5}{|c|}{ Preconception women (Estimated number) } & \multicolumn{5}{|c|}{ Currently pregnant women (Estimated number) } \\
\hline & Percent & $+/-\mathrm{CI}$ & $1000 \mathrm{~s}$ & $+/-\mathrm{CI}$ & $\mathrm{N}$ & $\overline{\text { Percent }}$ & $+/-\mathrm{CI}$ & $1000 \mathrm{~s}$ & $+/-\mathrm{CI}$ & $\mathrm{N}$ \\
\hline Total & $54.5^{*}$ & 3.7 & 1,024 & 94 & 2286 & 32.0 & 3.1 & 835 & 101 & 2949 \\
\hline No health plan/insurance & 63.4 & 11.7 & 222 & 52 & 328 & 52.7 & 10.3 & 156 & 50 & 303 \\
\hline Others & $52.5^{*}$ & 3.7 & 802 & 79 & 1957 & 29.4 & 3.2 & 679 & 88 & 2646 \\
\hline No personal doctor & $64.1^{*}$ & 9.0 & 278 & 59 & 448 & 46.3 & 9.2 & 234 & 73 & 467 \\
\hline Others & $51.6^{*}$ & 3.9 & 745 & 73 & 1837 & 28.6 & 2.9 & 601 & 71 & 2482 \\
\hline
\end{tabular}

Note. $95 \%$ confidence interval $=$ Percent $+/-\mathrm{CI}$ and Estimated number $+/-\mathrm{CI}$. Estimated number is the estimated number of women in the category with one of the risk factors.

${ }^{a}$ Any of 3 risk factors: frequent drinking, current smoker, no HIV test.

*difference between preconception and pregnant women significant, $p<.05$. 
more of these 3 risk factors, which represents approximately 1 million preconception women (Table 3); 32.0 percent of pregnant women had one or more of these risks, which is equivalent to 835,000 pregnant women. Table 3 also shows this composite risk factor by 2 measures of access to health care, whether the woman had a health plan or insurance and whether she had a personal physician. Pregnant women had a lower percent at risk in all categories except for those without health insurance. Among women without insurance, the percentage reporting risk was not significantly lower for pregnant women, compared with preconception women. This suggests that women with poorer access to health care, as indicated by no health insurance, are not as likely as others to adopt healthier behaviors when they become pregnant.

\section{Discussion}

The 2004 BRFSS provides an opportunity to measure the extent of risk behaviors on a relatively large sample of women who are planning to become pregnant. These nationallyrepresentative survey data indicate that major health risks were reported by substantial proportions of US women in the preconception period. The data suggest that among those at risk during preconception, the majority continued the risk into pregnancy. Women appear to respond to messages regarding behaviors that can improve pregnancy outcomes such as consuming of folic acid, and the reduction in smoking, alcohol consumption, but many remain unaware of the benefits of available interventions to prevent HIV transmission and birth defects. The fact that more than one-third of preconception and pregnant women were not aware of methods to prevent mother-to-child HIV transmission and did not know about the benefits of taking folic acid during pregnancy suggests that education and public information programs could have some effect in reducing risk behaviors.

Risk reduction appears to be lower among women who have no health insurance. Other data are not available for comparison of the overall burden of risk for adverse pregnancy outcomes among women in the preconception period or pregnancy. However, data on selected indicators presented here for pregnant women are comparable to the reported range from other studies [4, 6, 8-11].

The finding that the percentage who report some risk behaviors is significantly lower among pregnant women compared with women in the preconception period may indicate the desire of many pregnant women to adopt healthier behaviors to achieve the best possible outcome for their pregnancy. Examination of three well known risk factors for which interventions are available (frequent alcohol consumption, current smoking, or never having been tested for HIV) illustrates that risks for adverse pregnancy outcomes exist among more than half of women in the preconception period and among one-third of pregnant women. Nationwide, this represents approximately one million women in the preconception period and 835,000 pregnant women.

The data from the BRFSS are subject to potential errors associated with survey research such as exclusion from the sampling frame, non-response and reporting errors. BRFSS is a telephone-based survey and might not be representative of the small percentage of the population without telephones. The BRFSS methodology contains steps to ensure accurate estimation, including the use of weighting factors to compensate for non-response rates. In addition, estimates from BRFSS data have been found to be consistent with data from other surveys [5]. The preconception and pregnant women identified were distributed very similarly to women giving birth in 2004 by age group and race/ethnicity, providing support for the representativeness of the data. In this report, the reported risk behavior of women in the preconception stage and pregnant women have been compared as a proxy for the type of behavior change occurring between preconception and pregnancy, but other factors could affect the actual change in behavior among individual women.

For known risk factors, progress in efforts to reduce such risks among pregnant women has been slow. For example, as indicated in data collected on earlier rounds of the BRFSS, alcohol consumption among pregnant women has remained relatively steady over the past decade [10]. Even among those who reduce consumption of alcohol, tobacco, or drugs, post pregnancy relapse of such behaviors have been found to be very high [4]. Of the 17 maternal and infant health objectives included in the Healthy people 2010 objectives, progress has been made toward the target in 8 objectives [12]. Little positive progress has occurred in the areas of maternal death, fetal alcohol syndrome, and low birth weight.

To close the gap in reduction of poor maternal and child health outcomes, current maternal and child health initiatives should be complemented with alternate approaches, including more emphasis on preconception health promotion strategies among women of childbearing age. Recent experience in the prevention of alcohol-exposed pregnancy and prevention of neural tube defects through folic acid supplementation has shown that such efforts are useful. In 2003, the Project CHOICES Research Group reported the findings of a feasibility study designed to provide prevention counseling to women at high risk for an alcohol-exposed pregnancy [13]. Brief interventions were found to be effective in reducing hazardous alcohol use in adults in a number of well-controlled studies. Interventions generally consist of advice, feedback, goal setting, and follow-up for further assistance and support [14]. The use of a counseling style referred to as "motivational interviewing" has also proven to be effective in reducing problem drinking [15]. Results of the initial follow-up assessment, suggest that this approach 
can be effective among women of childbearing age [13], and a larger efficacy trial is now underway.

To summarize, our results were consistent with a high prevalence of risk factors in the preconception period, and a high degree continuation of risk into pregnancy. This underscores the need for new approaches to complement current prevention efforts. Given that women of childbearing age in the United States have on an average 6.4 visits to physicians each year [16], it may be feasible to inform them and engage them for interventions. Such contacts may provide an opportunity to assess and advise on many elements of health mentioned here. Our finding of high risk levels among women who had no insurance or no personal doctor strongly suggests that ensuring access to such services will remain the key determinant of maximum uptake of known intervention services. Medicaid and other funding programs can play an important role to address the access gap-whether during, before, or after pregnancy. Attempts to improve the health of would-be mothers not only improve pregnancy and infant health, but also help reduce the long-term impact of many risk factors on the women themselves.

\section{References}

1. National Center for Health Statistics. Health, United States, 2004. Hyattsville, MD: US Department of Health and Human Services, 2004.

2. Centers for Disease Control and Prevention. Deaths: Leading Causes for 2002. National Vital Statistics Reports 53 (17), 2005.

3. Kochanek KD, Martin JA. Supplemental analyses of recent trends in infant mortality. Centers for Disease Control and Prevention, National Center for Health Statistics. Accessed on August 18, 2004, at www.cdc.gov/nchs/products/pubs/pubd/hestats/infantmort/infantmort.htm.
4. Ebrahim SH, Gfroerer J. Pregnancy-related substance use in the United States during 1996-1998. Obstet Gynecol 2003;101:3749.

5. Balluz L, Ahluwalia IB, Murphy W, et al. Surveillance for Certain Health Behaviors Among Selected Local Areas-United States, Behavioral Risk Factor Surveillance System, 2002. MMWR 2004;53(SS05):1-100.

6. Williamson D, Serdula MK, Binkin NJ. Comparing the prevalence of smoking in pregnant and non-prengnant women, 1985 to 1986. JAMA 1989;261:70-4.

7. CDC. Revised recommendations for HIV screening of pregnant women. MMWR 2001;50(RR-19):59-86.

8. Serdula MK, Gillespie C, Kettel-Kahn L, et al. Trends in fruit and vegetable consumption among adults in the United States: Behavioral Risk Factor Surveillance System, 1994-2000. AJPH 2004;94:1014-8.

9. CDC. Use of vitamins containing folic acid among women of childbearing age-United States, 2004. MMWR 2004;53:847950.

10. CDC. Alcohol consumption among women who are pregnant or who might become pregnant-United States, 2002. MMWR 2004;53:1178-81.

11. CDC. Smoking during pregnancy-United States, 1990-2002. MMWR 2004;53:911-5.

12. US Department of Health and Human Services. Healthy People 2010. Washington, DC: US Department of Health and Human Services. 2000

13. Project CHOICES Research Group. Reducing the risk of alcohol-exposed pregnancies: a study of a motivational intervention in community settings. Pediatrics 2003;111(5):11315.

14. Whitlock EP, Polen M, Green CA, Orleans T, Klein J. Behavioral counseling interventions in primary care to reduce risky/harmful alcohol use by adults: a summary of the evidence for the U.S. preventive services task force. Annals of Internal Medicine 2004:140(7):557-68.

15. Miller WR, Rollnick S. Motivational interviewing: preparing people for change. 2nd ed. New York, NY: Guilford Press; 2002.

16. Adams PF, Morano MA. Current estimates from the National Health Interview Survey, 1994. National Center for Health Statistics. Vital Health Stat 1995;10:113. 